\title{
IMMUNOBIOLOGY
}

\section{The risk of hemophagocytic lymphohistiocytosis in Hermansky-Pudlak syndrome type 2}

\author{
Birthe Jessen, ${ }^{1}$ Sebastian F. N. Bode, ${ }^{1}$ Sandra Ammann, ${ }^{1}$ Subarna Chakravorty, ${ }^{2}$ Graham Davies, ${ }^{3}$ Jana Diestelhorst, ${ }^{4}$ \\ Melissa Frei-Jones, ${ }^{5}$ William A. Gahl, ${ }^{6}$ Bernadette R. Gochuico, ${ }^{6}$ Matthias Griese, ${ }^{4}$ Gillian Griffiths, ${ }^{7}$ Gritta Janka, ${ }^{8}$ \\ Christoph Klein, ${ }^{4}$ Tamara Kögl, ${ }^{9}$ Karin Kurnik, ${ }^{4}$ Kai Lehmberg, ${ }^{8}$ Andrea Maul-Pavicic, ${ }^{1}$ Andrew D. Mumford, ${ }^{10}$ David Pace, ${ }^{11}$ \\ Nima Parvaneh, ${ }^{12}$ Nima Rezaei, ${ }^{13}$ Geneviève de Saint Basile, ${ }^{14}$ Annette Schmitt-Graeff, ${ }^{15}$ Klaus Schwarz, ${ }^{16}$ \\ Gulsun T. Karasu, ${ }^{17}$ Barbara Zieger, ${ }^{18}$ Udo zur Stadt, ${ }^{19}$ Peter Aichele, ${ }^{9}$ and Stephan Ehl ${ }^{1}$
}

\begin{abstract}
${ }^{1}$ Centre of Chronic Immunodeficiency, University Medical Center Freiburg and University of Freiburg, Germany; ${ }^{2}$ Department of Medicine, Imperial College London, United Kingdom; ${ }^{3}$ Immunology Department, Great Ormond Street Hospital for Children, London, United Kingdom; ${ }^{4}$ Department of Pediatrics, Dr von Hauner Children's Hospital, Ludwig Maximilian University, Munich, Germany; ${ }^{5}$ The University of Texas Health Science Center San Antonio, San Antonio, TX; ${ }^{6}$ Section on Human Biochemical Genetics, Medical Genetics Branch, National Human Genome Research Institute, National Institutes of Health, Bethesda, MD; ${ }^{7}$ Cambridge Institute for Medical Research, Addenbrooke's Hospital, Cambridge, United Kingdom; ${ }^{8}$ University Medical Center Hamburg Eppendorf, Department of Pediatric Hematology and Oncology, Hamburg, Germany; ${ }^{9}$ Department of Immunology, Institute of Medical Microbiology and Hygiene, University of Freiburg, Germany; ${ }^{10}$ Bristol Heart Institute, University of Bristol, Bristol, United Kingdom; ${ }^{11}$ Department of Paediatrics, Mater Dei Hospital, Tal-Qroqq, Msida, Malta;

${ }^{12}$ Pediatric Infectious Diseases Research Center, Tehran University of Medical Sciences, Tehran, Iran; ${ }^{13}$ Research Center for Immunodeficiencies, Children's Medical Center, and Department of Immunology, School of Medicine, Tehran University of Medical Sciences, Tehran, Iran; ${ }^{14}$ INSERM U768, Université Paris Descartes, Hôpital Necker-Enfants Malades, Paris, France; ${ }^{15}$ Institute of Pathology, University Medical Center Freiburg, Freiburg, Germany; ${ }^{16}$ Institute for Transfusion Medicine, University of Ulm, Ulm, Germany; ${ }^{17}$ Division of Hematology and Medical Oncology, Akdeniz University Faculty of Medicine, Antalya, Turkey; ${ }^{18}$ Center for Pediatrics and Adolescent Medicine, University Medical Center Freiburg, Germany; and ${ }^{19}$ Center for Diagnostic, University Medical Center Hamburg Eppendorf, Hamburg, Germany
\end{abstract}

\section{Key Points}

- Hermansky-Pudlak syndrome type 2 confers a moderate risk for hemophagocytic lymphohistiocytosis.
Genetic disorders of lymphocyte cytotoxicity predispose patients to hemophagocytic lymphohistiocytosis (HLH). Reduced lymphocyte cytotoxicity has been demonstrated in Hermansky-Pudlak syndrome type 2 (HPS2), but only a single patient was reported who developed HLH. Because that patient also carried a potentially contributing heterozygous RAB27A mutation, the risk for HLH in HPS2 remains unclear. We analyzed susceptibility to HLH in the pearlmouse model of HPS2. After infection with lymphocytic choriomeningitis virus, pearl mice developed all key features of HLH, linked to impaired virus control caused by a moderate defect in CTL cytotoxicity in vivo. However, in contrast to perforin-deficient mice, the disease was transient, and all mice fully recovered and controlled the infection. An additional heterozygous Rab27a mutation did not aggravate the cytotoxicity defect or disease parameters. In the largest survey of 22 HPS2 patients covering 234 patient years, we identified only 1 additional patient with HLH and 2 with incomplete transient HLH-like episodes, although cytotoxicity or degranulation was impaired in all 16 patients tested. HPS2 confers a risk for HLH that is lower than in Griscelli or Chediak-Higashi syndrome, probably because of a milder defect in cytotoxicity. Preemptive hematopoietic stem cell transplantation does not appear justified in HPS2. (Blood. 2013;121(15):2943-2951)

\section{Introduction}

Hermansky-Pudlak syndrome type 2 (HPS2) is a rare autosomalrecessive disorder of lysosomal trafficking caused by mutations in the gene encoding the $\beta 3 \mathrm{~A}$ subunit of the adaptor protein 3 complex (AP-3). ${ }^{1,2}$ Patients with HPS2 display oculocutaneous albinism, bleeding diathesis, neutropenia, interstitial lung disease, pulmonary fibrosis, and suffer from recurrent infections. ${ }^{3}$ Immunological studies have shown that AP-3 deficiency leads to a defect in cytotoxicity of natural killer (NK) cells and cytotoxic T cells (CTL) as the result of impaired biogenesis and degranulation of perforin-containing lytic granules. ${ }^{4-7}$ Defective cytotoxicity caused by impaired degranulation also is observed in other disorders of lysosomal trafficking, such as Chediak-Higashi syndrome (CHS) and Griscelli syndrome type 2 (GS2), which are associated with albinism and in familial hemophagocytic lymphohistiocytosis (FHL) types 3-5. ${ }^{8,9}$ All of these diseases confer a high risk of developing hemophagocytic lymphohistiocytosis (HLH), a severe disorder of immune homeostasis that leads to a life-threatening hyperinflammatory disease. Hematopoetic stem cell transplantation (HSCT) can eliminate this risk and is therefore an important consideration at diagnosis. ${ }^{9,10}$

We have previously described 1 patient with HPS2 who developed severe HLH that was eventually lethal. ${ }^{5}$ This case, however,
Submitted October 22, 2012; accepted January 21, 2013. Prepublished online as Blood First Edition paper, February 12, 2013; DOI 10.1182/blood-2012-10463166.
The publication costs of this article were defrayed in part by page charge payment. Therefore, and solely to indicate this fact, this article is hereby marked "advertisement" in accordance with 18 USC section 1734.

B.J. and S.F.N.B. contributed equally to this work.

The online version of this article contains a data supplement. 
is the only one of severe HLH among 12 published patients with HPS2. ${ }^{2,5-7,11-14}$ Because this patient also carried a heterozygous $R A B 27 A$ mutation (causing GS2, if homozygous), it remains unclear whether HPS2 per se confers a risk for HLH or if the HLH observed in that patient was attributable to this additional mutation. Transient episodes of HLH were thereafter described in another patient with HPS $2,{ }^{6}$ and 1 additional patient had recurrent inflammatory episodes that did not fulfill all diagnostic criteria for $\mathrm{HLH}^{7}$ Overall, the risk of developing HLH in HPS2 remains incompletely defined. However, this risk is important for clinical decision-making because preventive treatment is available in the form of HSCT.

In this study, we used 2 approaches to address this problem. First, we analyzed pearl mice, who represent a mouse model for AP-3deficiency, in an experimental system known to provoke HLH in mouse strains with cytotoxicity defects. Although mice deficient in perforin, Rab27a, Lyst, Unc13d, or Syntaxin-11 do not develop HLH spontaneously, they develop all clinical features of the disease after infection with lymphocytic choriomeningitis virus (LCMV). ${ }^{15-20}$ This approach allowed us to compare the susceptibility to HLH of pearl mice with that of other cytotoxicity mutants after a defined viral trigger and to assess the impact of an additional heterozygous Rab27a mutation on the phenotype. Second, we performed a survey of all 12 published and 10 previously unreported HPS2 patients to document the clinical evolution of their disease and-if availablethe extent of their cytotoxicity defect. Our data show that the risk of developing HLH in HPS2 is relatively low because of a milder cytotoxicity defect. Considering the small risks and serious long-term pulmonary complications of the disease, which are not influenced by $\mathrm{HSCT}^{21}$ pre-emptive HSCT is probably not indicated in HPS2.

\section{Methods}

\section{Mice and virus}

C57BL/6 mice were purchased from Charles River Laboratories (Sulzfeld, Germany). Perforin-deficient (PKO) mice were originally generated on a C57BL/6 background by D. Kägi ${ }^{22}$ and were kindly provided by H. Pircher (IMMH, Freiburg, Germany). B6Pin.C3-Ap3bl $1^{p e} / \mathrm{J}$ (stock no. 003215; pearl $^{23}$ ) mice (backcross to C57BL/6) were purchased from The Jackson Laboratory (Bar Harbor, ME) and C57BL/6J-Rab27ash/J (ashen) mice backcrossed to C57BL/6 for 10 generations were kindly provided by G. de Saint Basile (INSERM, Paris, France). ${ }^{19}$ Pearl mice carrying a heterozygous Rab27a mutation were obtained by mating of pearl and ashen mice (pearl$a s h^{+/-}$). Mice were kept in an individual ventilated cage unit (BioZone, Kent, UK) and infected at the age of 6-12 weeks. The lymphocytic choriomeningitis virus WE (LCMV-WE) was provided by H. Pircher (IMMH, Freiburg, Germany). LCMV was grown on MC57G fibroblasts and stored at $-80^{\circ} \mathrm{C}$ until use. Mice were injected intravenously with 200 plaque-forming units (pfu) or $10^{4} \mathrm{pfu}$ as indicated. A focus-forming assay was used as described to quantify virus in organs from infected mice. ${ }^{24}$

\section{HLH biomarkers in mice}

Ferritin serum levels were measured by use of the Roche Modular Analytics Evo. Levels of sCD25 were analyzed with the mouse IL-2Ralpha DuoSet kit (R\&D Systems) according to the instructions of the manufacturer. The interferon (IFN)- $\gamma$ enzyme-linked immunosorbent assay was performed as described previously. ${ }^{17}$ Blood cell counts were determined with the Sysmex KX-21 hematology analyzer.

\section{Lymphocyte phenotyping and intracellular cytokine staining}

For flow cytometry, the following antibodies were used: CD3e (145-2C11; ebioscience), CD8a (53-6.7; BD), CD107a (1D4B; ebioscience), NK1.1
(PK136; BD), IFN- $\gamma$ (XMG1.2; BD), tumor necrosis factor- $\alpha$ (TNF- $\alpha$ : MP6-XT22; BD), KLRG1 (2F1; eBioscience), CD127 (A7R34; eBioscience), CD44 (IM7; eBioscience), and CD62L (MEL-14; eBioscience). To analyze the intracellular IFN- $\gamma$ or TNF- $\alpha$ production of CTL, spleen cells were restimulated with gp33 peptide (PolyPeptide, France) for 3 hours in the presence of monensin (BD). After surface staining, cells were fixed, permeabilized, and stained intracellularly for the respective cytokines.

\section{Degranulation and cytotoxicity assays}

To analyze NK-cell degranulation, mice were injected intraperitoneally with $200 \mu \mathrm{g}$ of polyinosinic acid/polycytidylic acid (poly (I:C); Sigma-Aldrich). One day later, spleen cells were restimulated for 2 hours with the NK-sensitive YAC-1 target cells or medium as a control in the presence of anti-CD107a antibody. Degranulation of NK1.1 $1^{+} \mathrm{CD}^{-} \mathrm{NK}$ cells was determined by flow cytometry. NK-cell cytotoxicity was determined by a 5-hour incubation of spleen cells with ${ }^{51}$ chromium-loaded YAC-1 target cells. Quantification of NK cells was performed by flow cytometry to calculate the NK-cell/target ratio. Surface expression of CD107a and intracellular IFN- $\gamma$ expression of $\mathrm{CD} 8{ }^{+} \mathrm{CD} 3^{+}$CTL were determined by flow cytometry. CTL cytotoxicity was determined in a 5-hour ${ }^{51}$ chromium-release assay by incubating spleen cells as effectors with either EL-4 cells loaded with gp33 or LCMV-infected MC57G cells as targets. ${ }^{25}$ To calculate the CTL/target ratio, CD8 T cells were quantified by antibody staining.

\section{Adoptive transfer experiments}

Splenic CD8 T cells from mice that had been infected with LCMV 8 days earlier were magnetic-activated cell sorting (MACS)-purified with use of the MACS CD8 ${ }^{+}$T Cell Isolation Kit II (Miltenyi Biotec). Purity was $>90 \%$ in all experiments. A total of $2 \times 10^{6}$ purified CD8 T cells were transfused into C57BL/6 mice that had been infected with $10^{4}$ pfu LCMV 10 hours before, and after additional 18 hours, splenic virus titers were determined. ${ }^{26}$

\section{Histology}

Organs were fixed with $4 \%$ formaldehyde and embedded in paraffin. Sectioning, staining, and analysis were performed as described previously. ${ }^{17}$

\section{Patients}

We performed a Pubmed search for "Hermansky-Pudlak Syndrome," "Hermansky-Pudlak syndrome type 2," "Adaptor protein 3," and "AP3B1" to identify all published HPS2 patients. We also contacted colleagues involved in the diagnosis of primary immunodeficiencies and HLH to identify additional patients. Material from 4 patients with HPS2 had been referred to the CCI Advanced Diagnostics Unit for functional studies. We contacted the corresponding authors of all relevant publications and all referring physicians to obtain current clinical information. Written informed consent for immunologic evaluation was obtained from the patients or their legal guardians according to the guidelines of the Declaration of Helsinki, as approved by the institutional review board at the University of Freiburg. NK-cell and CTL cytotoxicity and degranulation assays were performed according to local protocols. ${ }^{27}$

\section{Statistical analysis}

Tests were performed with the GaphPad InStat software version 3.06. The comparison between data were evaluated with a one-way analysis of variance with post-test. Differences were considered significant at a $P<.05$.

\section{Results}

\section{Pearl mice develop transient HLH after LCMV infection}

To evaluate whether AP-3-deficient mice are at risk of developing HLH, we infected pearl mice with 200 pfu of LCMV-WE 
Figure 1. Pearl mice develop transient HLH after LCMV infection independent of the presence of an additional Rab27a mutation. C57BL/6, pearl, pearl$\mathrm{ash}^{+/-}$, ashen, and $P K O$ mice were infected intravenously with 200 pfu of LCMV-WE, and (A) body weight and (B) temperature were monitored daily. Dashed lines indicate the detection limit. (C) Blood counts were analyzed on d8 after infection. The white line indicates the mean, and the gray area indicates the range of values of naïve pearl mice. D8 serum levels of (D) ferritin, (E) sCD25, and (F) IFN- $\gamma$ are shown. Graphs show pooled data of 2 independent experiments with 3 4 mice per group except for ashen mice, in which results from 3 mice obtained in 2 independent experiments are shown. n.s. indicates not significant $(P>$ .05); ${ }^{\star} P<.05 ;{ }^{\star \star} P<.01 ;{ }^{\star \star \star} P<.001$.
A

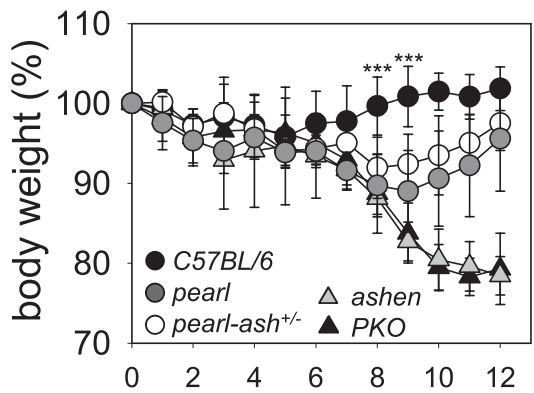
time after infection [days]

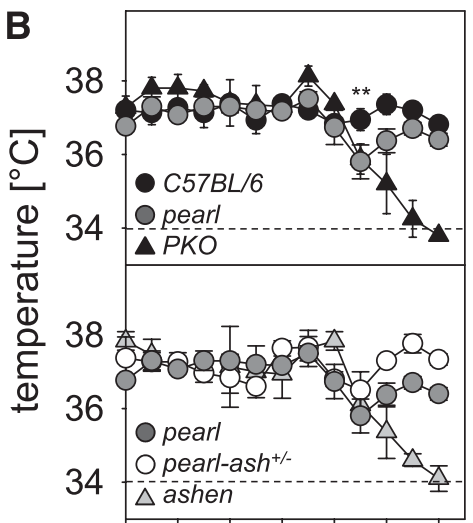

$\begin{array}{llllllll}0 & 2 & 4 & 6 & 8 & 10 & 12\end{array}$

time after infection [days]

C
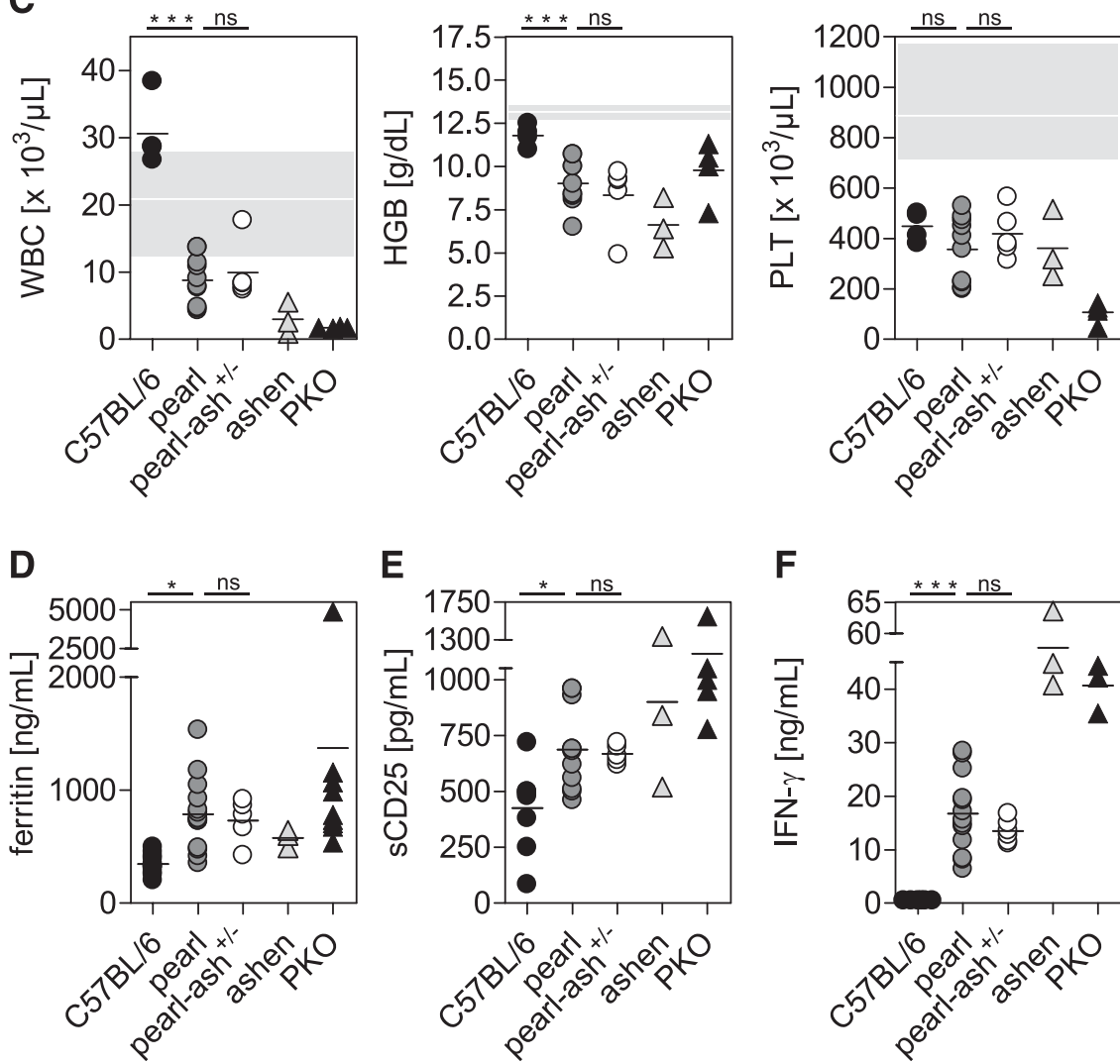

intravenously. Infection with LCMV has previously been shown to trigger HLH in a number of mouse strains representing models for human defects in lymphocyte cytotoxicity. ${ }^{15-19}$ To model the genetic situation of the single HPS2 patient who developed lethal HLH, ${ }^{5}$ we also infected pearl mice carrying an additional heterozygous Rab27a mutation (pearl-ash ${ }^{+/-}$). Homozygous Rab27a-deficient mice (ashen $)^{19}$ and $P K O^{15,18}$ were included as disease controls, and wild-type C57BL/6 mice served as negative controls. Because viral infections per se can cause a number of features of HLH in mice and humans, we applied the full set of HLH diagnostic criteria ${ }^{28}$ to the LCMV-infected mice with one exception: a decrease in ear temperature, reflecting circulatory centralization, was counted as fever. Thus, mice were considered to undergo HLH, if at least 5 of the 8 human diagnostic criteria were fulfilled. Elevation of serum levels of IFN- $\gamma$ and elevation of glutamic-pyruvic transaminase (GPT) and lactate dehydrogenase (LDH), which are also characteristic of human HLH, also were assessed and taken as supporting evidence.

After infection with LCMV, wild-type mice showed no changes in body weight and temperature, whereas ashen and PKO mice developed a significant decrease in body weight and temperature until the end of the experiment (d12). Pearl mice and pearl-ash ${ }^{+/-}$ mice also displayed weight loss and a decrease in body temperature starting 6 days after infection but eventually recovered starting at d9 (Figure 1A-B). At the time point of the most obvious disease (d8), wild-type mice showed leukocytosis, whereas pearl and pearl-ash ${ }^{+/-}$mice had leukopenia that was even more pronounced in ashen and PKO mice (Figure 1C). Pearl, pearl-ash ${ }^{+-}$, ashen, 
A
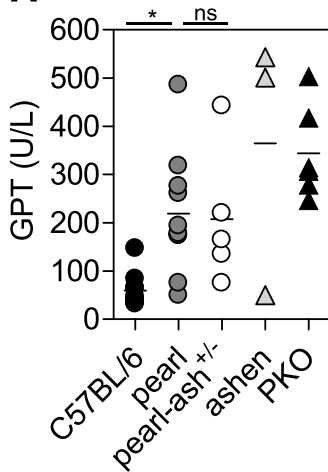
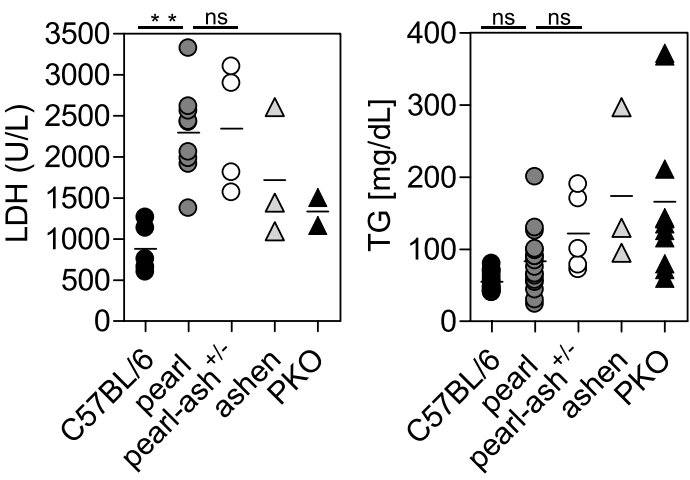

B

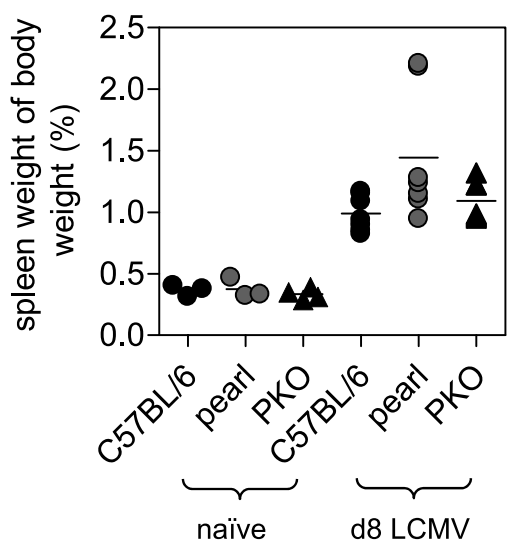

C
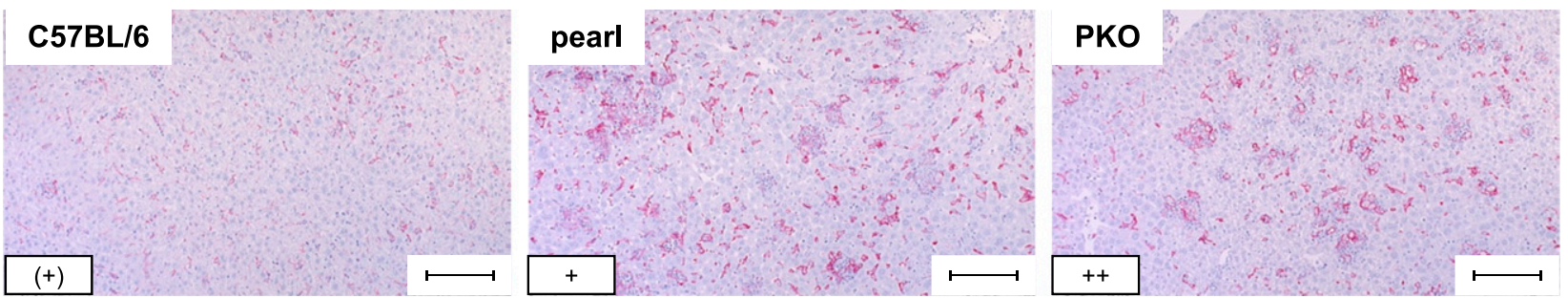

Figure 2. Liver disease and histopathological alterations in pearl mice undergoing LCMV-induced $\mathrm{HLH}$. C57BL/6, pearl, pearl-ash ${ }^{+/-}$, ashen, and $P K O$ mice were infected with LCMV-WE and 8 days later, serum levels of (A) GPT, LDH, and triglycerides (TG) were determined. (B) Splenomegaly was analyzed by calculating spleen weight in percent of body weight. (C) Representative liver sections stained with anti-F4/80 (original magnification, 10 $\times$ ) obtained at day 8 after infection. Lower left inset: semiquantitative analysis of hemophagocyting macrophages as assessed in 10 high-power visual fields $(40 \times)$ per mouse. $(+)$ indicates rare; + , few; and ++ , frequent. Scale bars represent $100 \mu \mathrm{m}$. n.s. indicates not significant $(P>.05) ;{ }^{\star} P<.05 ;{ }^{\star \star} P<.01$.

and $P K O$ mice showed anemia and all groups, including wild-type mice, developed thrombocytopenia (Figure 1C). Serum levels of ferritin (Figure 1D), sCD25 (Figure 1E), and IFN- $\gamma$ (Figure 1F) were elevated in all mutant mice, with the greatest values found in ashen and $P K O$ mice. Splenomegaly was observed in all groups, including wild-type mice, whereas triglycerides were increased in $P K O$ but not in pearl mice (Figure 2A-B), all mutant, but not wild-type mice showed significant elevation of GPT and LDH as well as histopathological evidence of hemophagocytosis (Figure 2C). Finally, splenic NK cells from pearl mice showed impaired degranulation and cytotoxicity in response to NKsensitive YAC-1 target cells 24 hours after injection of poly(I:C), although the response of individual mice was highly variable (Figure 3).

In summary, pearl mice developed 7 of 8 HLH criteria on $\mathrm{d} 8$ after LCMV infection. However, the abnormalities in some parameters
A

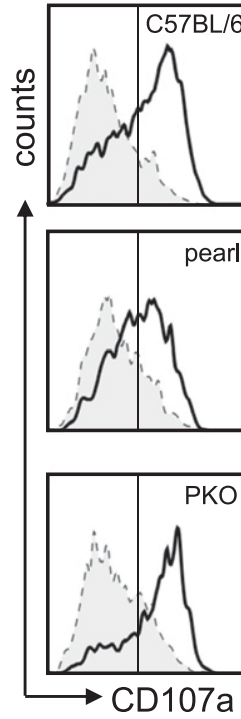

B

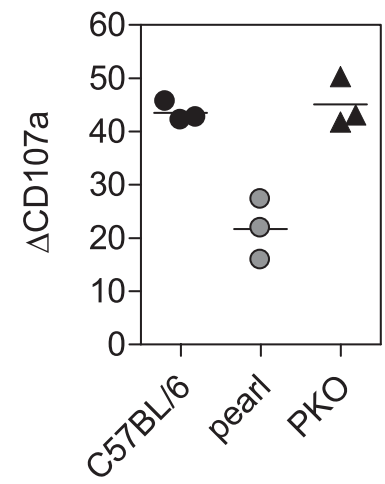

C

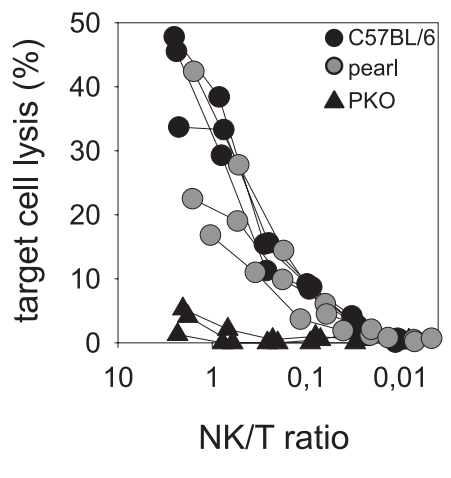

Figure 3. Impaired NK-cell function in pearl mice. C57BL/6, pearl, and PKO mice were injected intraperitoneally with $200 \mu \mathrm{g}$ of poly (I:C). At 24 hours later, spleen cells were restimulated for 2 hours with YAC-1 target cells or medium as a control in the presence of anti-CD107a antibody (A-B). (A) Representative histograms of $\mathrm{NK} 1.1^{+} \mathrm{CD}^{-} \mathrm{NK}$ cells are shown. Dashed line indicates medium control; solid line indicates restimulation with YAC-1 cells. (B) Degranulation is shown as increase of CD107a expression on $\mathrm{NK} 1.1^{+} \mathrm{CD}^{-}$cells $(\Delta \mathrm{CD} 107 \mathrm{a})$ after restimulation with YAC-1 cells compared with medium control. (C) NK-cell cytotoxicity was determined in a 5 -hour ${ }^{51}$ chromium release assay on YAC-1 target cells. Quantification of NK cells was performed by flow cytometry. Representative data for 3 independent experiments with 3-5 mice/group are shown. 

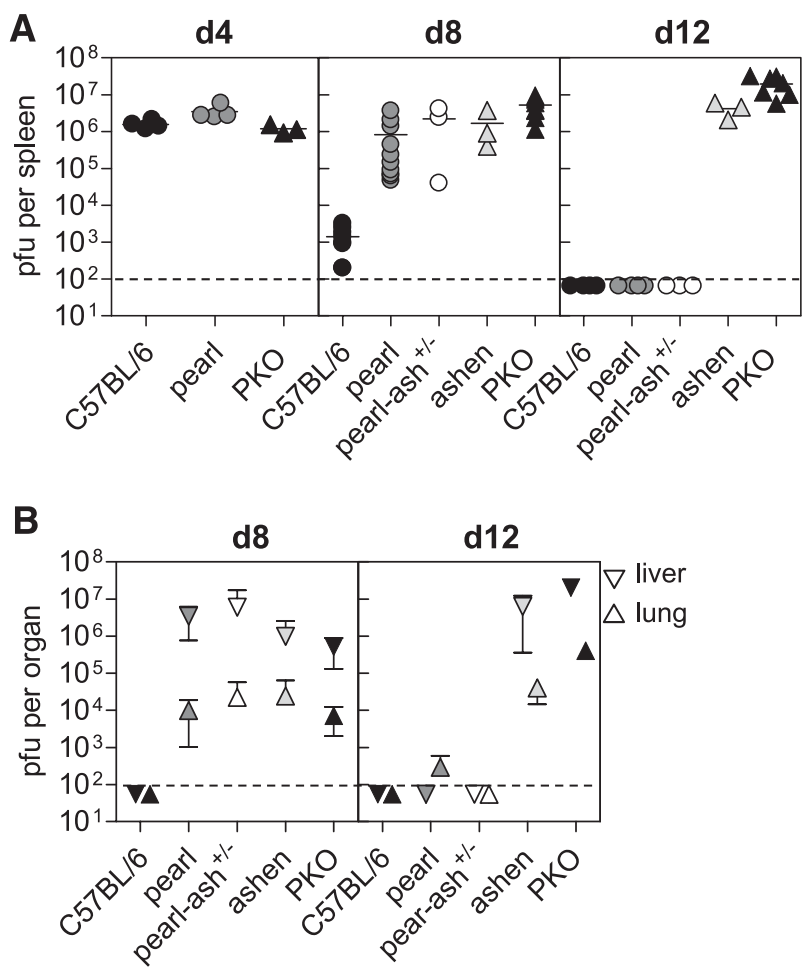

Figure 4. Pearl mice show a delay in virus control. C57BL/6, pearl, pearl-ash ${ }^{+/-}$, ashen, and PKO mice were infected with 200 pfu of LCMV-WE and (A) virus titer in spleens were determined on days 4, 8 , and 12 after infection. (B) Days 8 and 12 virus titers are shown for lung and liver. The means and the SDs are indicated. Graphs show pooled data of 2 independent experiments with 3-4 mice per group. For ashen mice data of 3 mice obtained in 2 different experiments are shown. The dashed lines indicate the detection limit.

were less severe than in ashen or $P K O$ mice and pearl mice recovered from the disease without any treatment. Thus, pearl mice had regained body weight and normalized ear temperature, leukocyte counts, and serum levels of ferritin, $\mathrm{sCD} 25$, and IFN- $\gamma$ but retained some thrombocytopenia and splenomegaly at d12 after infection (supplemental Figure 1; see the Blood Web site). Of note, the additional heterozygous Rab27a mutation did not enhance any of the disease parameters in pearl mice.

\section{Recovery from HLH in pearl mice is associated with eventual virus elimination}

In our recent study in mouse models of CHS, we found that HLH manifestations were associated with the persistence of LCMV. ${ }^{17}$ We therefore analyzed virus elimination kinetics in the different strains in spleen, liver, and lung. Splenic viral titers were comparable in wild-type, pearl, and $P K O$ mice at day 4 after infection (Figure 4A). However, although wild-type mice had almost eliminated the virus by day 8 after infection, pearl and pearl-ash ${ }^{+/-}$ mice retained a virus load that was similar to that of ashen and $P K O$ mice (Figure 4A). At this time point, spread of LCMV to lungs and livers was observed in all mutant mice (Figure 4B). Interestingly, determination of viral titers on day 12 after infection revealed that pearl and pearl-ash ${ }^{+/-}$mice had eliminated LCMV below detection limit in all investigated organs, whereas ashen and $P K O$ mice developed long-term virus persistence (Figure 4A-B). Thus, the presence of HLH correlated with loss of virus control in these cytotoxicity mutants as well.
Pearl CTL have a defect in degranulation and cytotoxicity that contributes to delayed virus control

Pearl mice show a number of immunological defects, including lack of NKT cells, defects in antigen presentation, and impaired cytotoxicity in vitro. ${ }^{29-33}$ To determine whether reduced cytotoxicity was a critical factor for the impairment of virus control and development of HLH, we analyzed the capacity of pearl CTL to degranulate and to lyse LCMV-infected target cells in vitro and to control LCMV infection in vivo. Eight days after infection with LCMV, pearl CTL showed a moderate but significant reduction in degranulation in response to the immunodominant LCMV gp33 peptide compared with wild-type C57BL/6 mice (Figure 5A-B). This degranulation defect was reflected in a reduced ex vivo cytotoxicity on LCMV-infected target cells (Figure 5D, left), which was more pronounced when CTL activity was analyzed on target cells loaded with a limiting concentration of gp33 peptide $\left(10^{-10} \mathrm{M}\right.$; Figure $5 \mathrm{D}$, right). We then transfused MACS-purified activated CTL from pearl or C57BL/6 control mice into mice that had been infected with $10^{4}$ pfu LCMV 10 hours earlier and determined virus titers in the spleen after additional 18 hours. In this short-term protection assay, virus control depends on perforin-mediated cytotoxicity. ${ }^{26}$ While transfusion of activated wild-type CTL could clear the infection in this short-term assay, pearl CTL were not able to fully control the virus (Figure 5C). These data indicated that the CTL cytotoxicity defect contributed to the lack of virus control.

\section{Pearl CTL are phenotypically and functionally altered on d8 after LCMV infection but regain functionality after virus elimination}

We have recently shown in a mouse model of FHL-4 that HLH in cytotoxicity mutant mice is accompanied by characteristic phenotypic and functional alterations of virus-specific $\mathrm{CD}^{+} \mathrm{T}$ cells. ${ }^{20}$ We therefore analyzed the production of intracellular IFN- $\gamma$ upon gp33 stimulation of spleen cells obtained on days 8 and 12 after LCMV infection. On day 8 after infection, the total frequency of IFN$\gamma$-producing CTL was similar (data not shown), but the relative frequency of IFN- $\gamma^{\text {high }} \mathrm{CD} 8^{+} \mathrm{T}$ cells was lower in pearl and $\mathrm{PKO}$ compared with control mice (Figure 6A). Moreover, the fraction of cells expressing TNF- $\alpha$ was reduced in the 2 mutant strains compared with control mice (Figure 6B). However, at day 12 the staining pattern for both cytokines returned to normal in pearl mice, whereas $P K O$ CTL remained less responsive to antigen stimulation (Figure 6A-B). A similar pattern was observed when we analyzed the differentiation phenotype of $\mathrm{CD}^{+} \mathrm{T}$ cells. On day 8, pearl CTL resembled $P K O$ $\mathrm{CTL}$ with a reduced fraction of $\mathrm{KLRG} 1^{+} \mathrm{CD} 127^{-}$cells (Figure 6C) and of $\mathrm{CD} 44^{\mathrm{hi}} \mathrm{CD} 62^{\text {lo }}$ cells (Figure 6D), whereas at day 12 after infection the phenotype of pearl CTL resembled that of wild-type CTL (Figure 6C-D). Thus, the altered phenotype was linked to virus control and was transient in pearl CTL, whereas it persisted in PKO CTL.

\section{HPS2 patients have a moderate risk of developing HLH}

The findings in the pearl mouse model suggested that because of a less-severe defect in cytotoxicity, the risk of developing HLH is lower and episodes are less severe than in other cytotoxicity mutants. To assess the risk of HLH in human HPS2 patients, we performed a survey of all 12 so-far published patients with HPS2 and 10 additional, previously unreported patients (Table 1). For 5 patients, we had to rely on the information given in the published report ${ }^{2,7,14}$; for all other patients, a 2012 update on the clinical evolution of the disease was provided. The mean age at last follow-up was 10.6 years 
A

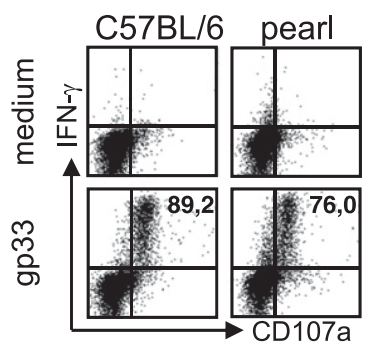

B

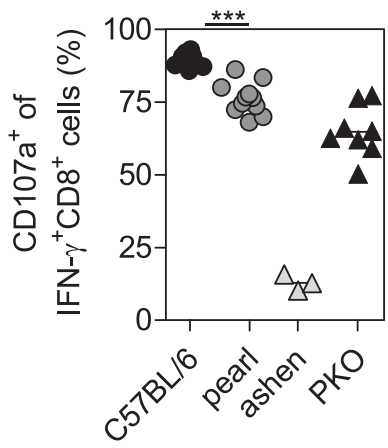

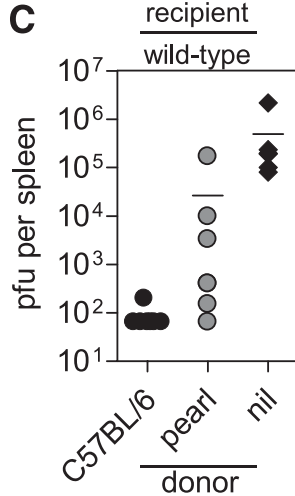

Figure 5. CTL of pearl mice are impaired in degranulation and cytotoxicity. CTL from C57BL/6, pearl, ashen, and PKO mice were analyzed 8 days after infection with 200 pfu of LCMV-WE. Degranulation of LCMV-specific CTL was analyzed after in vitro restimulation with the gp33 peptide. (A) Representative fluorescence-activated cell-sorting plots gated on $\mathrm{CD}^{+} \mathrm{CD}^{+}$lymphocytes are shown. Numbers indicate the percentage of $\mathrm{CD} 107 \mathrm{a}^{+}$cells among gp33-specific IFN- $\gamma^{+}$CTL. (B) Frequencies of CD107a ${ }^{+}$cells of IFN$\gamma^{+} \mathrm{CD}^{+} \mathrm{T}$ cells were analyzed. Pooled data from 3 independent experiments are shown. ${ }^{* * *} P<.001$ (C) Wild-type mice were infected with $10^{4}$ pfu LCMV. Then, 10 hours later they were adoptively transfused with $2 \times$ $10^{6}$ isolated day 8 splenic CTL of either wild-type or pearl mice and after additional 18-hour splenic virus titers were analyzed. Pooled data from 2 independent experiments with 3-4 mice per group are shown. The dashed line indicates the detection limit. Nil indicates without transfer. (D) Ex vivo cytotoxicity was tested in a 5 -hour ${ }^{51}$ chromiumrelease assay on either LCMVinfected MC57 target cells (left) or gp33 peptide-loaded EL-4 target cells (right). Results from 1 of 2 independent experiments with 3 mice per group are shown

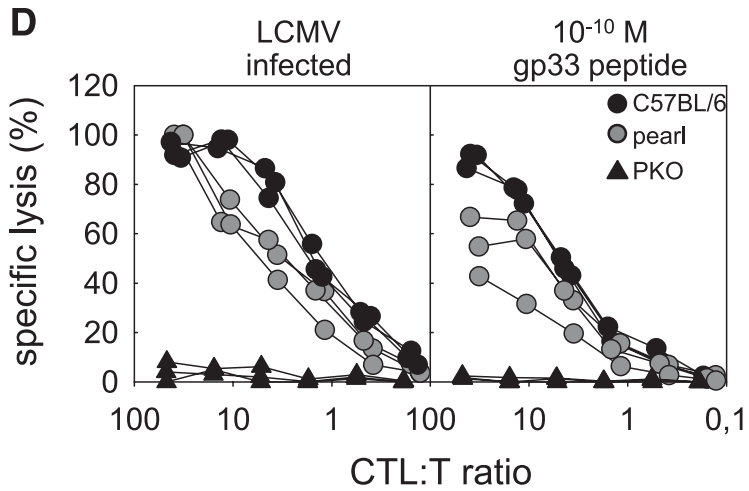

(range, 1.25-30 years) and the overall observation covered 234 patient years. There were 5 sibling pairs, 6 patients had compound heterozygous mutations, and 16 had homozygous mutations. All but 1 patient had neutropenia; 12 received granulocyte-stimulating colony factor (G-CSF) therapy. Eighteen patients had recurrent bacterial and viral infections, most of which affecting the respiratory
A
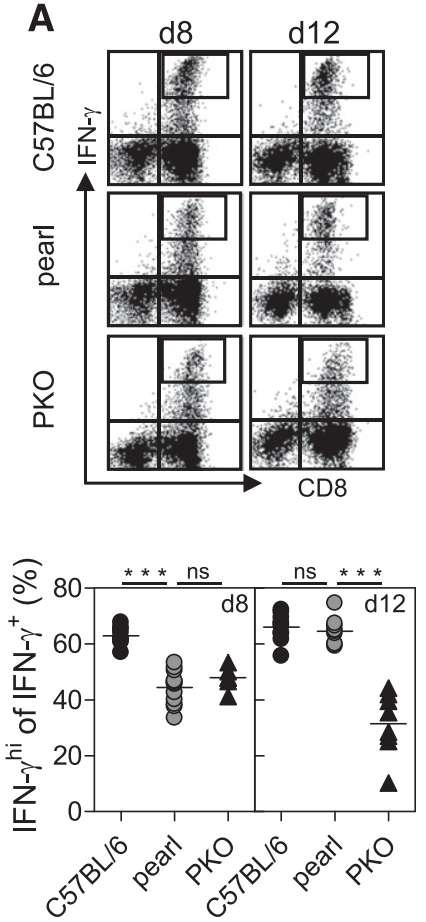

B

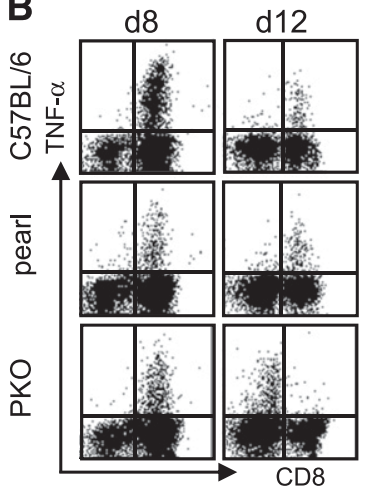

C
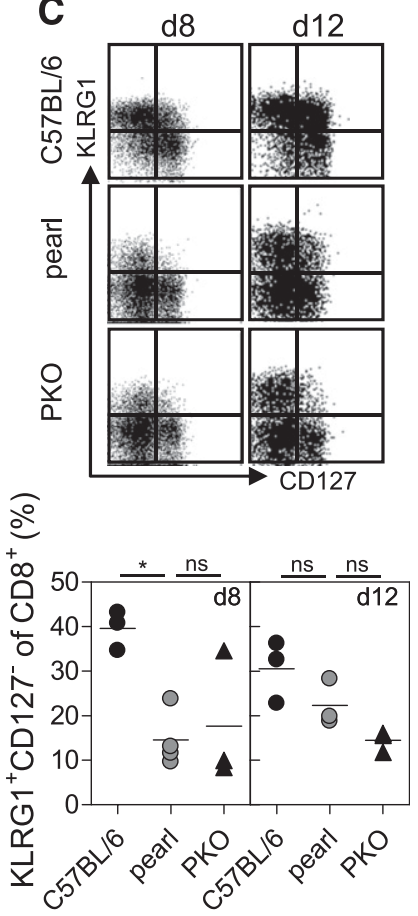
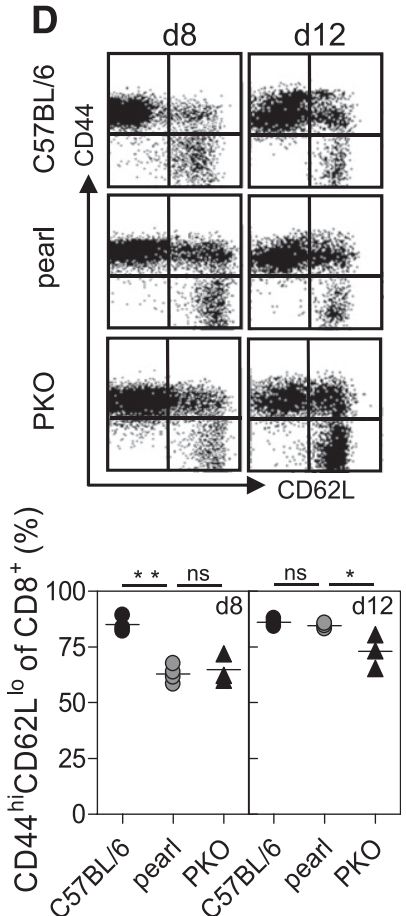

Figure 6. Function and phenotype of pearl CTL at different time points after infection. (A, B) Cytokine production of $C 57 B L / 6$, pearl, and $P K O C T L$ was analyzed by flow cytometry on days 8 and 12 after infection with 200 pfu of LCMV. CTL were stained for (A) IFN- $\gamma$ and (B) TNF- $\alpha$ production after peptide stimulation in vitro. Representative FACS plots are shown at the top and (A) frequencies of IFN- $\gamma^{\text {hi }}$ cells among IFN- $\gamma^{+}$CD8 T cells and (B) frequencies of TNF- $\alpha^{+}$cells among CD8 T cells are shown at the bottom. Surface expression of (C) KLRG1 and CD127 and (D) CD44 and CD62L on CTL are depicted as representative FACS plots (top) and frequencies are shown (bottom). Representative data from 1 of 2 independent experiments with 3-4 mice per group are shown. For IFN- $\gamma$ and TNF- $\alpha$ (day 8 ) pooled data from two independent experiments are shown. n.s. indicates not significant $(P>.05) ;{ }^{\star} P<.05 ;{ }^{\star \star} P<.01 ;{ }^{\star \star \star} P<.001$. 
Table 1. Patients with HPS2

\begin{tabular}{|c|c|c|c|c|c|c|c|c|}
\hline \multirow{2}{*}{$\begin{array}{l}\text { Patient } \\
\text { no./ID } \\
\text { (reference) }^{\star}\end{array}$} & \multirow[b]{2}{*}{ AP3B1 mutation } & \multirow{2}{*}{$\begin{array}{l}\text { Age at } \\
\text { last visit, } \\
\text { years }\end{array}$} & \multicolumn{5}{|c|}{ Clinical features } & \multirow{2}{*}{$\begin{array}{l}\text { NK or CTL } \\
\text { cytotoxicity and/or } \\
\text { degranulation }\end{array}$} \\
\hline & & & Neutropenia & Bleeding & $\begin{array}{l}\text { Recurrent } \\
\text { infections }\end{array}$ & $\begin{array}{l}\text { Pulmonary } \\
\text { fibrosis }\end{array}$ & HLH & \\
\hline $1(7)$ & $\begin{array}{c}\text { EX10 } \\
\text { c.1063_1064delCAinsTATCAATATC; } \\
\text { p.Q355fsX360 } \\
\text { EX16 c. } 1789 \_1790 \text { insA; } \\
\text { p.1597fsX608 }\end{array}$ & 7 & $+(\mathrm{G}-\mathrm{CSF})$ & - & + & - & - & Impaired \\
\hline 27 & $\begin{array}{c}\text { EX10 } \\
\text { c.1063_1064delCAinsTATCAATATC; } \\
\text { p.Q355fsX360 } \\
\text { EX16 c. } 1789 \_1790 \text { insA; } \\
\text { p.1597fsX608 }\end{array}$ & 4 & $+(\mathrm{G}-\mathrm{CSF})$ & + & + & + & One incomplete episode & Impaired \\
\hline P2 (6) & EX19 c.2078_2165del; p.E693fsX706 & 6 & + & - & - & - & - & Impaired \\
\hline P3 (6) & EX2 c.153_156delAGAG p.E52fsX63 & 7.2 & $+(\mathrm{G}-\mathrm{CSF})$ & - & + & $(+)$ & Two incomplete episodes & Impaired \\
\hline FR5t & EX27 c.3222_3223delTG; p.E1075fs & $5 \ddagger$ & + & ++ & + & ++ & - & Impaired \\
\hline FR1378 & EX27 c.3222_3223delTG; p.E1075fs & 13 & $+(\mathrm{G}-\mathrm{CSF})$ & $(+)$ & $(+)$ & + & - & Impaired \\
\hline $29(14)$ & EX15 g.del8172bp; p.del491-550 & 18 & $+(\mathrm{G}-\mathrm{CSF})$ & - & + & n.a. & - & n.a. \\
\hline $30(14)$ & EX15 g.del8172bp; p.del491-550 & 21 & $+(\mathrm{G}-\mathrm{CSF})$ & - & + & n.a. & - & n.a. \\
\hline $40(2)$ & $\begin{array}{c}\text { EX11-12 c.1166_1228del; } \\
\text { p.del390-410 } \\
\text { EX16 c.1739T>G; p.L580R }\end{array}$ & 22 & + & + & + & + & $\begin{array}{l}- \\
-\end{array}$ & n.a. \\
\hline $42(2)$ & $\begin{array}{c}\text { EX11-12 c.1166_1228del; } \\
\text { p.del390-410 } \\
\text { EX16 c.1739T>G; p.L580R }\end{array}$ & 27 & + & + & + & + & - & n.a. \\
\hline FR50 & Del Exon 16 & $16 \ddagger$ & $+(\mathrm{G}-\mathrm{CSF})$ & - & + & ++ & - & Impaired \\
\hline $87(13)$ & $\begin{array}{l}\text { Ex15 c. } 1525 C>T ; p . R 509 \times \\
\text { Ex18 c. } 1975 G>T ; p . E 659 \times\end{array}$ & 17 & + & + & + & + & - & Impaired \\
\hline FR393 (5) & EX8 c.904A>T; p.R302× & $3 \neq$ & + & + & + & - & One lethal episode & Impaired \\
\hline FR1315 & EX18 c.2041G>T; p.E681× & 2 & $+(\mathrm{G}-\mathrm{CSF})$ & - & - & - & Two episodes & Impaired \\
\hline$A, P(11)$ & IVS10+5G $>A$ & 6 & $+(\mathrm{G}-\mathrm{CSF})$ & + & + & + & - & n.a. \\
\hline$C, B$ & EX1 c60_delG; p.L20fsX41 & $1,25 \ddagger$ & + & n.a. & + & n.a. & - & Impaired \\
\hline C, A & EX1 c60_delG; p.L20fsX41 & $2 \ddagger$ & $+(\mathrm{G}-\mathrm{CSF})$ & n.a. & + & n.a. & - & Impaired \\
\hline L, M & EX23 c.2770_delC; p.L924fsX926 & 2.5 & $+(\mathrm{G}-\mathrm{CSF})$ & $(+)$ & $(+)$ & - & - & Impaired \\
\hline W, M & EX23 c.2770_delC; p.L924fsX926 & 11 & $+(\mathrm{G}-\mathrm{CSF})$ & $(+)$ & $(+)$ & - & - & Impaired \\
\hline$M, Z$ & EX4 c.205T>C p.L102P & 11 & + & + & - & - & - & n.a. \\
\hline n.a. (12) & $\begin{array}{c}\text { IVS14+6T }>C \\
\text { EX15 c.1619insG; } p . A 541 \text { fsX565 }\end{array}$ & 30 & - & - & - & - & - & Impaired \\
\hline n.a. & $\operatorname{inv}(5)(q 14.1)$ & 2.25 & + & - & + & - & - & Impaired \\
\hline
\end{tabular}

N.a. indicates not available.

${ }^{*}$ Patient ID in published report.

†Kurnik et al, manuscript submitted 2013.

‡Deceased.

tract. Eleven of 20 patients, where this information was available, had clinically relevant episodes of bleeding, and 9 of 18 patients had evidence of lung fibrosis.

Apart from the reported patient with lethal $\mathrm{HLH},{ }^{5}$ one other patient could be identified who developed 2 transient episodes fulfilling 5 of $8 \mathrm{HLH}$ criteria. Two further patients had spontaneously resolving episodes of fever and cytopenia in association with varicella and CMV infections that did not fulfill the diagnostic criteria but may have represented incomplete manifestations of HLH. ${ }^{6,7}$ Of the 5 reported deaths in HPS2 patients, 1 was from HLH, 2 from pneumonia in the context of lung fibrosis and 2 after accidents. In 4 patients without any HLH-related symptoms, asymptomatic seroconversion to Epstein-Barr virus, a known strong trigger for HLH, was documented. At least one immunological test addressing NK-cell or CTL cytotoxicity, NK-cell, or CTL degranulation was available from 16 patients. The experimental protocols including local reference values varied from laboratory to laboratory. However, abnormal findings in at least one of these tests were reported in all 16 patients. We also restudied 3 patients with our previously evaluated standardized panel of diagnostic tests. ${ }^{27}$ In these in vitro tests, they showed degranulation abnormalities that were similar to that of patients with CHS or GS2 (supplemental Figure 2). Overall, despite a significant degranulation and cytotoxicity defect, human HPS2 appears to confer only a limited risk for developing HLH.

\section{Discussion}

HPS2 is a rare genetic disorder of lysosomal trafficking with clinical manifestations in several organ systems..$^{25-7,11-14}$ As for other subtypes of HPS, affected patients have a diathesis toward bleeding as the result of a platelet storage pool deficiency ${ }^{34}$ and a risk of lung fibrosis as the result of aberrant repair mechanisms of the lung caused by lysosomal mistrafficking. ${ }^{21}$ In addition, however, patients with HPS2 have significant immunological problems, manifesting mainly as chronic neutropenia and a susceptibility to infections. This is in part explained by chronic neutropenia reflecting a role for AP-3 in neutrophil development, ${ }^{3,35,36}$ but HPS2 also involves other immunological abnormalities, such as an impaired development of NKT cells, ${ }^{14}$ impaired TLR recruitment to phagosomes, and impaired MHC-II 
presentation of antigens internalized by phagocytosis. ${ }^{32,37,38}$ Finally, lytic granule exocytosis depends upon AP-3, leading to deficiencies of NK-cell and CTL mediated cytotoxicity in HPS2. ${ }^{5-7,12}$

Because of limited patient numbers, little is known about the prognosis of affected patients, which renders treatment decisions difficult. Life-threatening events may encompass episodes of serious bleeding, severe infections, or respiratory failure caused by progressive pulmonary fibrosis. Moreover, the defect in lymphocyte cytotoxicity can predispose patients to HLH. ${ }^{5,8,39}$ Because HSCT can prevent the risk of HLH in other genetic disorders of cytotoxicity, can correct the platelet defect, and mitigate the increased susceptibility to infection, HSCT is a relevant consideration for HPS2 patients. ${ }^{9,10,40,41}$ However, this treatment carries its own risks and may not affect lung fibrosis, ${ }^{42,43}$ so it must be carefully balanced against the natural history of the disease. In this study we combined experimental studies in a mouse model of HPS2 with observations in the largest reported cohort of 22 HPS2 patients to evaluate the extent to which AP-3 deficiency predisposes to HLH. We also examined the effect of other disease manifestations on prognosis.

Pearl mice carry a 793-bp tandem duplication in the Ap3bl gene altering the reading frame and truncating the protein 130 amino acids from the $\mathrm{C}$-terminus. ${ }^{23}$ There are conflicting data concerning residual protein expression in these mice, ${ }^{34,44}$ and we cannot fully exclude the possibility that some functional AP-3 remains. Nevertheless, the mice recapitulate the platelet storage pool deficiency, ${ }^{34}$ the pigment dilution, ${ }^{45}$ and the lung pathology. ${ }^{46,47}$ They also show impaired NKT cell development ${ }^{29,30}$ and impaired TLR mediated type I IFN responses. ${ }^{32,37,38}$ In one previous report authors analyzed NK-cell cytotoxicity in pearl mice and found that it was not significantly altered. $^{48}$ In our study, we found a mild NK cell and CTL degranulation defect leading to impaired cytotoxicity in pearl mice. This was less pronounced than in PKO mice or in Lyst-mutant souris mice. ${ }^{17}$ Nevertheless, there was a clear impact of the defect on CTL-mediated control of LCMV infection. However, in contrast to PKO mice, pearl mice were eventually able to clear the virus from all organs.

The authors of previous studies have shown that lymphocyte cytotoxicity is also significantly impaired in HPS2 patients. Although initial studies who used T-cell lines found a severe defect, ${ }^{12}$ subsequent studies demonstrated significant residual activity., ${ }^{5,6} \mathrm{Ex}$ vivo NK-cell cytotoxicity was reduced or absent in all reported patients. ${ }^{5,7}$ In the patients collected for our study, different protocols were used to analyze CTL or NK-cell degranulation and/or cytotoxicity, but at least one abnormal test result was reported in all 16 patients analyzed. Of note, CTL cytotoxicity, which is a less sensitive assay, was normal in 3 patients despite abnormal NK-cell degranulation tests. This pattern has been observed previously in patients with variants of CHS associated with a low risk of $\mathrm{HLH}^{17}$

We investigated to what extent the cytotoxicity defect in HPS2 predisposes to HLH. Indeed, as for other cytotoxicity mutants, infection of pearl mice with LCMV-induced HLH as assessed by the 8 criteria that define the human disease. ${ }^{28}$ However, in contrast to other strains, the pearl mice recovered spontaneously without any treatment once the virus was controlled. Pearl mice thus represent the first mouse model for transient HLH, represented by a disease course that can also be observed in FHL patients with milder cytotoxicity defects as the result of hypomorphic mutations. ${ }^{49,50}$ The recovery of these mice from HLH after the virus is controlled indicates that the pathogenic uncontrolled immune response inducing HLH is not autonomous but that continuous antigen stimulation is a key factor in the maintenance of the disease. This finding was also reflected by a more detailed analysis of disease-inducing LCMV-specific T cells. At the peak of clinical disease, pearl mice showed phenotypic and functional alterations of CTL that were indistinguishable from those of PKO mice. However, once the virus was controlled, these functional and phenotypic changes disappeared, whereas they were maintained in PKO mice, where virus and disease manifestations persisted.

Our clinical observations indicate that the risk for HPS2 patients of developing HLH is clearly present but much lower than for patients with Griscelli syndrome type 2, CHS, or FHL. Considering our findings in pearl mice, this is likely attributable to the milder defect in cytotoxicity, allowing delayed, but eventually full control of the dysregulated immune response. The fact that pearl mice carrying an additional heterozygous Rab27a mutation showed the same disease course as pearl mice without that mutation suggests that the severe course of HLH in our previously reported HPS2 patient was not caused by that additional mutation.

The prognosis of the patients in our cohort was not only determined by HLH alone. Of the 3 patients who died of disease manifestations, 2 died of pneumonia in the context of lung fibrosis. In fact, 9 of 18 patients had evidence of lung fibrosis. This disease manifestation is caused by impaired pulmonary repair mechanism attributable to disturbed vesicle trafficking that may not be corrected by bone marrow transplantation. The neutropenia was responsive to G-CSF in all patients, significantly reducing the risk of infections. Only a single patient had significant recurrent bleeding episodes. Taken together, this study suggests that pre-emptive HSCT is not indicated in this patient group, as it is in GS2 and some patients with CHS. ${ }^{17}$ Nevertheless, HPS2 patients and their treating physicians must be aware of the fact that there is a relevant risk of HLH and must be alert to the early signs of this potentially lifethreatening disease manifestation.

\section{Acknowledgments}

The authors thank Nadja Goos for excellent technical support.

This study was supported by the Bundesministerium für Bildung und Forschung (BMBF 01 EO 0803), the Thyssen Stiftung, the EU FP7 (CURE-HLH), and in part by the Intramural Research Program of the National Human Genome Research Institute, National Institutes of Health.

\section{Authorship}

Contribution: B.J., S.F.N.B., and S.E. designed the study and prepared the manuscript; B.J. performed experiments in mice; S.F.N.B., S.A., and A.M.-P. collected and analyzed patient data; S.C., G.D., J.D., M.F.-J., W.A.G., B.R.G., M.G., G.G., G.J., C.K., K.K., K.L., D.P., N.P., G.d.S.B., G.T.K., N.R., and B.Z. recruited patients for the study and contributed the clinical data; A.S.-G. performed histological analysis; A.D.M., K.S., and U.z.S. performed genetic analysis of HPS2 patients; and T.K. and P.A. gave technical support and conceptual advice.

Conflict-of-interest disclosure: The authors declare no competing financial interests

Correspondence: Stephan Ehl, Center of Chronic Immunodeficiency, Breisacher Str. 117, 79106 Freiburg, Germany; e-mail: stephan.ehl@uniklinik-freiburg.de. 


\section{References}

1. Hermansky F, Pudlak $P$. Albinism associated with hemorrhagic diathesis and unusual pigmented reticular cells in the bone marrow: report of two cases with histochemical studies. Blood. 1959; 14(2):162-169.

2. Shotelersuk V, Dell'Angelica EC, Hartnell L, et al. A new variant of Hermansky-Pudlak syndrome due to mutations in a gene responsible for vesicle formation. Am J Med. 2000;108(5):423-427.

3. Badolato R, Parolini S. Novel insights from adaptor protein 3 complex deficiency. J Allergy Clin Immunol. 2007;120(4):735-741;quiz 742-733.

4. Clark R, Griffiths GM. Lytic granules, secretory lysosomes and disease. Curr Opin Immunol. 2003;15(5):516-521.

5. Enders A, Zieger B, Schwarz K, et al. Lethal hemophagocytic lymphohistiocytosis in Hermansky-Pudlak syndrome type II. Blood. 2006;108(1):81-87.

6. Wenham M, Grieve S, Cummins M, et al. Two patients with Hermansky Pudlak syndrome type 2 and novel mutations in AP3B1. Haematologica. 2010;95(2):333-337.

7. Fontana S, Parolini S, Vermi W, et al. Innate immunity defects in Hermansky-Pudlak type 2 syndrome. Blood. 2006;107(12):4857-4864.

8. Bode SF, Lehmberg K, Maul-Pavicic A, et al Recent advances in the diagnosis and treatment of hemophagocytic lymphohistiocytosis. Arthritis Res Ther. 2012;14(3):213.

9. Janka GE. Hemophagocytic syndromes. Blood Rev. 2007;21(5):245-253.

10. Jordan MB, Allen CE, Weitzman S, et al. How I treat hemophagocytic lymphohistiocytosis. Blood. 2011;118(15):4041-4052.

11. Chiang PW, Spector E, Thomas M, et al. Nove mutation causing Hermansky-Pudlak Syndrome Type 2. Pediatr Blood Cancer. 2010;55(7):1438.

12. Clark RH, Stinchcombe JC, Day A, et al. Adaptor protein 3-dependent microtubule-mediated movement of lytic granules to the immunological synapse. Nat Immunol. 2003;4(11):1111-1120.

13. Huizing M, Scher CD, Strovel E, et al. Nonsense mutations in ADTB3A cause complete deficiency of the beta3A subunit of adaptor complex-3 and severe Hermansky-Pudlak syndrome type 2. Pediatr Res. 2002;51(2):150-158.

14. Jung J, Bohn G, Allroth A, et al. Identification of a homozygous deletion in the AP3B1 gene causing Hermansky-Pudlak syndrome, type 2. Blood. 2006;108(1):362-369.

15. Binder D, van den Broek MF, Kägi D, et al Aplastic anemia rescued by exhaustion of cytokine-secreting CD8 $+\mathrm{T}$ cells in persistent infection with lymphocytic choriomeningitis virus. $J$ Exp Med. 1998;187(11):1903-1920.

16. Crozat K, Hoebe K, Ugolini S, et al. Jinx, an MCMV susceptibility phenotype caused by disruption of Unc13d: a mouse model of type 3 familial hemophagocytic lymphohistiocytosis. $J$ Exp Med. 2007;204(4):853-863.

17. Jessen B, Maul-Pavicic A, Ufheil $H$, et al. Subtle differences in CTL cytotoxicity determine susceptibility to hemophagocytic lymphohistiocytosis in mice and humans with Chediak-Higashi syndrome. Blood. 2011;118(17): 4620-4629.

18. Jordan MB, Hildeman D, Kappler J, et al An animal model of hemophagocytic lymphohistiocytosis (HLH): CD8 + T cells and interferon gamma are essential for the disorder. Blood. 2004;104(3):735-743.

19. Pachlopnik Schmid J, Ho CH, Diana J, et al. A Griscelli syndrome type 2 murine model of hemophagocytic lymphohistiocytosis (HLH). Eur J Immunol. 2008;38(11):3219-3225.

20. Kogl T, Muller J, Jessen B, et al. Hemophagocytic lymphohistiocytosis (HLH) in Syntaxin-11 deficient mice: T-cell exhaustion limits fatal disease. Blood. 2013;121(4):604-613.

21. Gochuico BR, Huizing M, Golas GA, et al. Interstitial lung disease and pulmonary fibrosis in Hermansky-Pudlak syndrome type 2, an adaptor protein-3 complex disease. Mol Med. 2012;18(1) 56-64.

22. Kägi D, Ledermann B, Bürki K, et al. Cytotoxicity mediated by $T$ cells and natural killer cells is greatly impaired in perforin-deficient mice. Nature. 1994;369(6475):31-37.

23. Feng $L$, Seymour $A B$, Jiang $S$, et al. The beta3A subunit gene (Ap3b1) of the AP-3 adaptor complex is altered in the mouse hypopigmentation mutant pearl, a model for Hermansky-Pudlak syndrome and night blindness. Hum Mol Genet. 1999;8(2):323-330.

24. Battegay M, Cooper S, Althage A, et al. Quantification of lymphocytic choriomeningitis virus with an immunological focus assay in 24- or 96-well plates. J Virol Methods. 1991;33(1-2): 191-198.

25. Pircher H, Moskophidis D, Rohrer U, et al. Viral escape by selection of cytotoxic $T$ cell-resistan virus variants in vivo. Nature. 1990;346(6285): 629-633.

26. Ehl S, Klenerman $\mathrm{P}$, Aichele $\mathrm{P}$, et al. A functional and kinetic comparison of antiviral effector and memory cytotoxic T lymphocyte populations in vivo and in vitro. Eur J Immunol. 1997;27(12): 3404-3413.

27. Bryceson YT, Pende D, Maul-Pavicic A, et al. A prospective evaluation of degranulation assays in the rapid diagnosis of familial hemophagocytic syndromes. Blood. 2012;119(12):2754-2763.

28. Henter JI, Horne A, Aricó M, et al. HLH-2004: Diagnostic and therapeutic guidelines for hemophagocytic lymphohistiocytosis. Pediatr Blood Cancer. 2007;48(2):124-131.

29. Cernadas $M$, Sugita $M$, van der Wel $N$, et al Lysosomal localization of murine CD1d mediated by AP-3 is necessary for NK T cell development. J Immunol. 2003;171(8):4149-4155.

30. Elewaut D, Lawton AP, Nagarajan NA, et al. The adaptor protein AP-3 is required for CD1d-mediated antigen presentation of glycosphingolipids and development of Valpha14 NKT cells. J Exp Med. 2003;198(8):1133-1146.

31. Sugita M, Cao X, Watts GF, et al. Failure of trafficking and antigen presentation by CD1 in AP. 3-deficient cells. Immunity. 2002;16(5):697-706.

32. Mantegazza AR, Guttentag SH, El-Benna J, et al. Adaptor protein-3 in dendritic cells facilitates phagosomal toll-like receptor signaling and antigen presentation to CD4(+) T cells. Immunity. 2012;36(5):782-794

33. Li W, Rusiniak ME, Chintala S, et al. Murine Hermansky-Pudlak syndrome genes: regulators of lysosome-related organelles. Bioessays. 2004; 26(6):616-628

34. Zhen L, Jiang S, Feng L, et al. Abnorma expression and subcellular distribution of subunit proteins of the AP-3 adaptor complex lead to platelet storage pool deficiency in the pearl mouse. Blood. 1999;94(1):146-155.

35. Benson KF, Li FQ, Person RE, et al. Mutations associated with neutropenia in dogs and humans disrupt intracellular transport of neutrophil elastase. Nat Genet. 2003;35(1):90-96.

36. Massullo P, Druhan LJ, Bunnell BA, et al. Aberrant subcellular targeting of the G185R neutrophil elastase mutant associated with severe congenital neutropenia induces premature apoptosis of differentiating promyelocytes. Blood. 2005;105(9):3397-3404.

37. Blasius AL, Arnold CN, Georgel P, et al. Slc15a4, AP-3, and Hermansky-Pudlak syndrome proteins are required for Toll-like receptor signaling in plasmacytoid dendritic cells. Proc Natl Acad Sci U S A. 2010;107(46):19973-19978.

38. Sasai M, Linehan MM, Iwasaki A. Bifurcation of Toll-like receptor 9 signaling by adaptor protein 3 . Science. 2010;329(5998):1530-1534.

39. Fischer A, Latour S, de Saint Basile G. Genetic defects affecting lymphocyte cytotoxicity. Curr Opin Immunol. 2007;19(3):348-353

40. Eapen M, DeLaat CA, Baker KS, et al. Hematopoietic cell transplantation for ChediakHigashi syndrome. Bone Marrow Transplant. 2007;39(7):411-415

41. Pachlopnik Schmid J, Moshous D, Boddaert N, et al. Hematopoietic stem cell transplantation in Griscelli syndrome type 2: a single-center report on 10 patients. Blood. 2009;114(1):211-218.

42. McGarry MP, Borchers M, Novak EK, et al. Pulmonary pathologies in pallid mice result from nonhematopoietic defects. Exp Mol Pathol. 2002; 72(3):213-220.

43. Young LR, Gulleman PM, Bridges JP, et al. The alveolar epithelium determines susceptibility to lung fibrosis in Hermansky-Pudlak syndrome. Am J Respir Crit Care Med. 2012;186(10):1014-1024.

44. Peden AA, Rudge RE, Lui WW, et al. Assembly and function of AP-3 complexes in cells expressing mutant subunits. J Cell Biol. 2002 156(2):327-336.

45. Balkema GW, Mangini NJ, Pinto LH. Discrete visual defects in pearl mutant mice. Science. 1983;219(4588):1085-1087

46. Young LR, Borchers MT, Allen HL, et al. Lungrestricted macrophage activation in the pear mouse model of Hermansky-Pudlak syndrome. $J$ Immunol. 2006;176(7):4361-4368.

47. Young LR, Pasula R, Gulleman PM, et al. Susceptibility of Hermansky-Pudlak mice to bleomycin-induced type II cell apoptosis and fibrosis. Am J Respir Cell Mol Biol. 2007;37(1): 67-74.

48. Clark EA, Shultz LD, Pollack SB. Mutations in mice that influence natural killer (NK) cell activity. Immunogenetics. 1981;12(5-6):601-613.

49. Meeths M, Chiang SC, Wood SM, et al. Familial hemophagocytic lymphohistiocytosis type 3 (FHL3) caused by deep intronic mutation and (FHersion in UNC13D. Blood. 2011;118(22): 5783-5793.

50. Zhang $\mathrm{K}$, Jordan MB, Marsh RA, et al. Hypomorphic mutations in PRF1, MUNC13-4, and STXBP2 are associated with adult-onset familial HLH. Blood. 2011;118(22):5794-5798. 